\title{
Immune checkpoint inhibitor-related pneumonitis induced by camrelizumab: a case report and review of literature
}

\author{
Ling Li, Anqi Lou, Junxian Yu \\ Department of Pharmacy, Beijing Friendship Hospital, Capital Medical University, Beijing, China \\ Correspondence to: Junxian Yu. Beijing Friendship Hospital, Capital Medical University, Beijing 100050, China. Email: junxianyu@ccmu.edu.cn.
}

\begin{abstract}
Immune checkpoint inhibitors (ICIs) are new agents that are efficacious in a variety of cancers. However, they are associated with immune-related adverse events due to activated immune system. Among them, checkpoint inhibitor pneumonitis (CIP) deserves more special attentions, because diagnosis and therapy are still challengeable. camrelizumab is a programmed cell death 1 (PD-1) inhibitor that was developed by Jiangsu Hengrui Medicine Co. CIP that is induced by camrelizumab was rarely reported. We described a case that a patient developed CIP 12 days later after one dose of camrelizumab. A 60-yearold man with advanced esophageal squamous cell carcinoma received 6 cycles of Tislelizumab/placebo, capecitabine and cisplatin first. Owing to the poor control of the disease, the patient used Nimotuzumab and docetaxel on April 3, 2020 and April 24, 2020, respectively. Later, he obtained the combination of $200 \mathrm{mg}$ of camrelizumab and $140 \mathrm{mg}$ of docetaxel regimen for once on May 14, 2020. After 12 days, he was diagnosed with CIP in Outpatient. Multiple ground glass opacities were revealed in bilateral lungs from routine CT examination. After giving $40 \mathrm{mg}$ of prednisolone orally once a day, his CIP improved. Meanwhile, camrelizumab was not used again. Teaching point: same as other PD-1 inhibitors, camrelizumab can also cause immune-related pneumonitis. Close observation, regular CT examination and timely corticosteroids intervention are essential.
\end{abstract}

Keywords: Checkpoint inhibitor pneumonitis (CIP); anti-programmed cell death (PD-1) blockade; immunerelated pneumonitis (IMP); camrelizumab; case report

Submitted Jan 05, 2021. Accepted for publication Mar 24, 2021.

doi: 10.21037/apm-21-23

View this article at: http://dx.doi.org/10.21037/apm-21-23

\section{Introduction}

The incidence of immune-related pneumonitis (IMP) caused by programmed cell death (PD-1) and programmed cell death ligand 1 (PD-L1) inhibitors is about 2.2\% (1). The incidence is not high, while the number of death due to IMP is considerable (2). The probability of IMP that is induced by PD-1 inhibitors is higher than other immune checkpoint inhibitors (ICIs). Compared with other pneumonitis, the occurrence of checkpoint inhibitor pneumonitis (CIP) is relatively late (3-7). The risks of CIP are still vague, but the occurrence of CIP may be closely related to smoking history, previous treatment (whether radiotherapy), combination therapy, the type of primary tumor, and baseline lung disease (8-10). The predictive biomarkers for CIP are also unclear. Some studies revealed that the increase in CXCR2, IL1ra and IL2ra is correlated with the development of CIP (11). At the same time, CIP patient has higher level of baseline peripheral-blood absolute eosinophil count (AEC) (12).

Camrelizumab is a novel humanized high-affinity IgG4kappa monoclonal antibody and it was approved for the treatment of patients with relapsed or refractory classical Hodgkin lymphoma. The most common adverse reactions $(\geq 10 \%)$ include cutaneous capillary endothelial hyperplasia, anemia, fever, fatigue, hypothyroidism, proteinuria, and cough $(13,14)$. There are few reports on pneumonitis caused by camrelizumab. We describe a case that a patient developed IMP 12 days after one dose of camrelizumab.

We present the following article in accordance with 


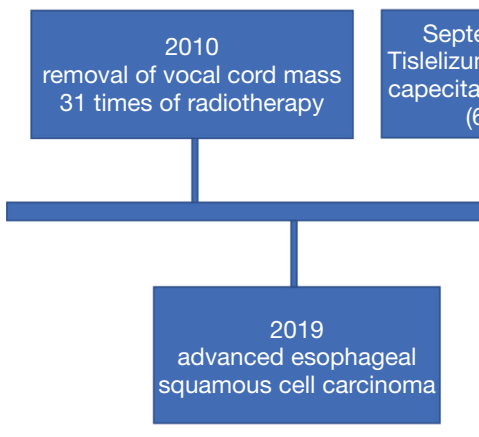

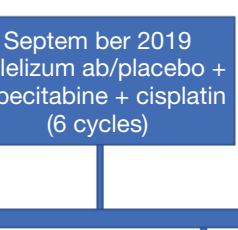

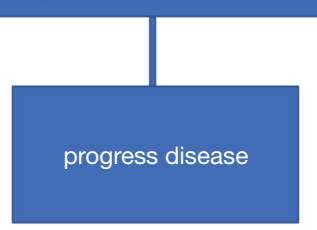

April 2020

nimotuzumab +

docetaxel ( 2 cycles)
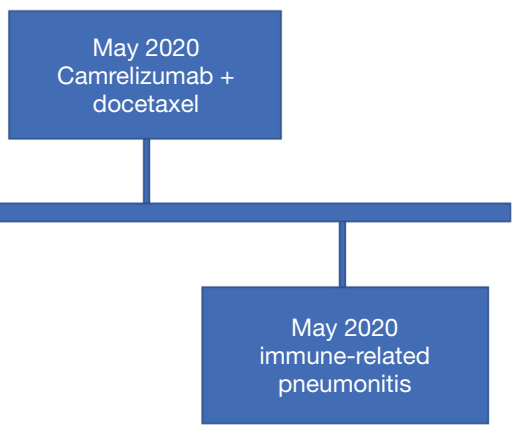

Figure 1 Timeline of disease and treatment.

the CARE reporting checklist (available at http://dx.doi. org/10.21037/apm-21-23).

\section{Case presentation}

The patient was a 60 -year-old male, $181 \mathrm{~cm}$ in height, $65 \mathrm{~kg}$ in weight, and $1.84 \mathrm{~m}^{2}$ in surface area. He has been smoking for 25 years, with the average of 20 cigarettes a day. Ten years ago, the patient underwent surgical resection for a mass on the vocal cord. Postoperative pathology suggested squamous epithelial dysplasia with precancerous lesions. In order to prevent malignant lesions, 31 times of radiotherapy were performed after surgery. In September 2019, he was diagnosed with advanced esophageal squamous cell carcinoma with an Eastern Cooperative Oncology Group Score (ECOG) of 1, with the stage of T4NxM1. Afterwards, he participated in a Phase III clinical trial: A phase 3 study on Tislelizumab (BGB-A317) in the combination with chemotherapy as the first-line treatment for unresectable, locally advanced, recurrent or metastatic esophageal squamous cell carcinoma. It is a randomized, placebo-controlled, and double-blind study. The patient was treated through chemotherapy (capecitabine $3.5 \mathrm{~g}$ day 1-15; cisplatin $109 \mathrm{mg} \mathrm{q} 3 \mathrm{w}$ ) and Tislelizumab/placebo $200 \mathrm{mg} \mathrm{q} 3 \mathrm{w}$ (Figure 1). After 6 cycles of treatment, he withdrew from the trial due to the development of the disease. The following treatment was started with the administration of nimotuzumab and docetaxel. He received the regimen for twice on 2 April 2020 and 24 April 2020, respectively.

The patient was admitted to the hospital on May 13 to further adjust the anticancer drug, and received $200 \mathrm{mg}$ of camrelizumab plus $140 \mathrm{mg}$ of docetaxel in the following day. However, after 12 days, multiple ground glass opacities were revealed in bilateral lungs from routine CT examination (Figure 2).

During the treatment of camrelizumab, the suffocation improved. The patient previously had coughs and hoarseness due to esophageal cancer, but it did not aggravate recently.

Routine blood examination: white blood cells (WBC): $8.38 \times 10^{9} / \mathrm{L}$, absolute neutrophils (GR): $7.04 \times 10^{9} / \mathrm{L}$, percentage of neutrophils (GR\%): $84.0 \%$, and C-reactive protein (CRP): $124.61 \mathrm{mg} / \mathrm{L}$. The patient's blood and sputum culture were negative. He was thought to be developed with IMP. First, the patient's CT findings matched the typical features of the IMP (15). Second, although the patient had experienced radiotherapy, the ground-glass opacities were not limited to prior radiation field and were outside the high radiation dose. The possibility of RRP is low (16). Third, some researches indicated that radiation history represents one of the high risk factors of IMP (17). Fourth, the tumor location of patient did not overlap with the area of pneumonitis, so the situation of tumor pseudo progression could be ruled out.

He received $40 \mathrm{mg}$ of Prednisolone per day orally. To prevent infection, the patient used fluconazole and moxifloxacin. Even with a significant radiographic improvement, camrelizumab was discontinued. In the follow-up treatment, the patient administered Paclitaxel for Injection (Albumin Bound), Oxaliplatin and Tegafur Gimeracil Oteracil Potassium Capsule and received 3 courses of treatment. The condition was assessed as SD. All procedures performed in studies involving human participants were in accordance with the ethical standards of the institutional and national research committees and with the Helsinki Declaration (as revised in 2013). Written informed consent was obtained from the patient. 

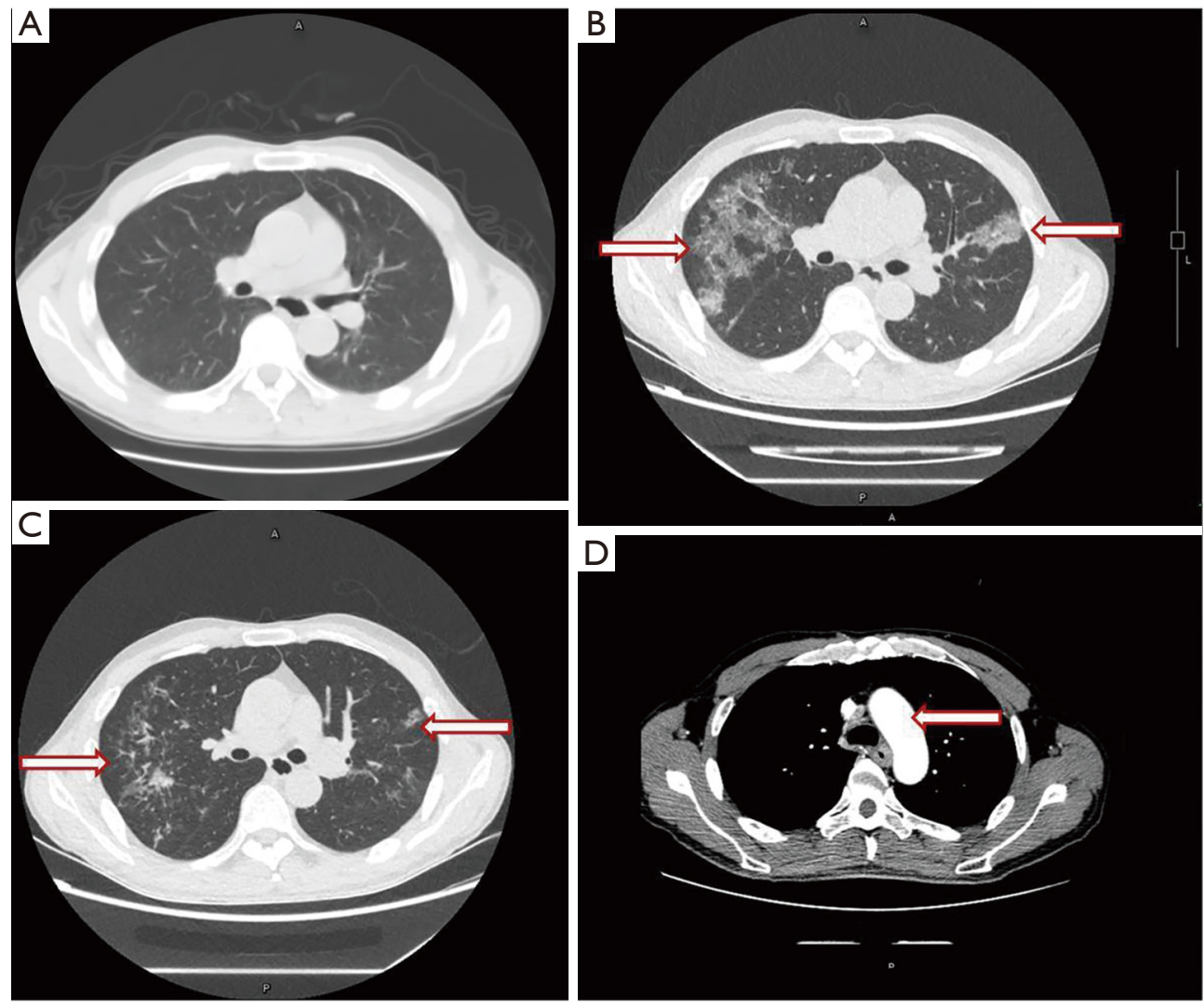

Figure 2 Chest CT of patient. (A) Before administration of camrelizumab; (B) after administration of camrelizumab; (C) after treatment; (D) image of patient's tumor. Arrows indicate inflammatory lesions and cancer lesion respectively.

\section{Discussion}

PD-1 is a receptor expressed by immune cells such as T cells, B Cells, and natural killer cells (18). Through PD1 pathway, immune response can be regulated to anticancer. There are currently eight PD-1/PD-L1 inhibitors on the market in China, namely atezolizumab, camrelizumab, durvalumab, nivolumab pembrolizumab, tislelizumab, toripalimab and sintilimab. The possible mechanisms of immune-related adverse reactions are as follows: PD-1/ PD-L1 inhibitors up-regulate cellular immune checkpoint molecules, which may result in the destruction of immune tolerance (19). There are similar or identical antigens in the affected tissues and the tumor tissues (20-23).

The most common symptoms of pneumonitis include dyspnea, cough, chest pain and fever. But some patients have no symptoms (24). Radiographic pattern can be manifested as cryptogenic organizing pneumonitis, nonspecific interstitial pneumonitis, hypersensitivity pneumonitis, acute interstitial pneumonitis and acute respiratory distress syndrome (25). If the patient meets the following three conditions: the history of ICIs, new pulmonary shadow in imageological examination and excluding pneumonitis caused by other circumstances, CIP can be diagnosed. Most patients get remission from pneumonitis, but those with grade 3 or higher pneumonitis or co-infection may have a poor prognosis. Early treatment with corticosteroids can reduce potentially life-threatening factors caused by pneumonitis. Depending on the severity of adverse reactions, the management methods are different (Table 1).

In this case, the patient obtained different treatments, we tended to think that it was due to the administration of camrelizumab for pneumonitis, while it could not rule out the efficiency of Tislelizumab. Immune-associated pneumonitis usually occurs after receiving 3-4 cycles of PD-1/PD-L1 inhibitors. However, the imaging of the lungs was normal after six cycles of Tislelizumab/placebo, including the dressing change process. Additionally, when the patient terminated camrelizumab, his pneumonitis 
Table 1 Management of immune-related pneumonitis

\begin{tabular}{|c|c|c|c|c|}
\hline & ESMO (26) & $\operatorname{NCCN}(27)$ & ASCO (28) & SITC (29) \\
\hline Symptom & $\begin{array}{l}\text { Radiographic changes } \\
\text { only: ground glass } \\
\text { change }\end{array}$ & $\begin{array}{l}\text { Asymptomatic; confined to } \\
\text { one lobe or less than } 25 \% \text { of } \\
\text { the lung parenchyma; clinical } \\
\text { or diagnostic observations } \\
\text { only }\end{array}$ & $\begin{array}{l}\text { Asymptomatic; confined to } \\
\text { one lobe of the lung or }<25 \% \\
\text { of lung parenchyma; clinical or } \\
\text { diagnostic observations only }\end{array}$ & $\begin{array}{l}\text { Asymptomatic; clinical or } \\
\text { diagnostic observations only }\end{array}$ \\
\hline Investigations & $\begin{array}{l}\text { High resolution } \mathrm{CT}+/- \\
\text { bronchoscopy and BAL } \\
\text { pending appearances }\end{array}$ & $\begin{array}{l}\text { Oxygen saturation; repeat } \\
\text { CT in } 4 \text { weeks or sooner } \\
\text { if clinically indicated for } \\
\text { worsening symptoms; pulse } \\
\text { oximetry reassess in } 1 \text { to } \\
2 \text { weeks }\end{array}$ & $\begin{array}{l}\text { Offer one repeat CT and } \\
\text { spirometry/DLCO in 3-4 weeks; } \\
\text { pulse oximetry; CXR }\end{array}$ & $\begin{array}{l}\text { Repeat CT at least every } \\
3 \text { weeks; oxygen saturation } \\
\text { every 2-3 days; weekly clinic } \\
\text { visits }\end{array}$ \\
\hline \multicolumn{5}{|l|}{ Grade 2} \\
\hline Symptom & $\begin{array}{l}\text { Mild/moderate new } \\
\text { symptoms }\end{array}$ & New/worsening symptoms & $\begin{array}{l}\text { Symptomatic; involves more } \\
\text { than one lobe of the lung or } \\
25-50 \% \text { of lung parenchyma; } \\
\text { medical intervention indicated; } \\
\text { limiting instrumental adl }\end{array}$ & $\begin{array}{l}\text { Symptomatic; limiting } \\
\text { instrumental adl; medical } \\
\text { intervention indicated }\end{array}$ \\
\hline PD-1 inhibitor & Withhold & Delay & $\begin{array}{l}\text { Hold until resolution to g1 or } \\
\text { less }\end{array}$ & Hold \\
\hline \multicolumn{5}{|l|}{ Grade 3 or 4} \\
\hline \multirow[t]{2}{*}{ Symptom } & G3: severe symptoms & $\begin{array}{l}\text { G3: severe symptoms; } \\
\text { involves all or more than } \\
50 \% \text { of the lung parenchyma; } \\
\text { limiting self-care ADL }\end{array}$ & $\begin{array}{l}\text { G3: severe symptoms; involves } \\
\text { all lung lobes or more than } \\
50 \% \text { of lung parenchyma; } \\
\text { limiting self-care ADL; oxygen } \\
\text { indicated }\end{array}$ & $\begin{array}{l}\text { G3: severe symptoms; } \\
\text { limiting self-care ADL; } \\
\text { oxygen indicated }\end{array}$ \\
\hline & G4: life-threatening & $\begin{array}{l}\text { G4: life-threatening; } \\
\text { impairment of respiratory } \\
\text { function }\end{array}$ & $\begin{array}{l}\text { G4: life-threatening; respiratory } \\
\text { compromise; urgent } \\
\text { intervention indicated }\end{array}$ & $\begin{array}{l}\text { G4: life-threatening; } \\
\text { respiratory compromise; } \\
\text { urgent intervention indicated }\end{array}$ \\
\hline
\end{tabular}

Table 1 (continued) 
Table 1 (continued)

\begin{tabular}{|c|c|c|c|c|}
\hline & ESMO (34) & $\operatorname{NCCN}(35)$ & ASCO (36) & SITC (37) \\
\hline
\end{tabular}

ESMO, European Society for Medical Oncology; NCCN, National Comprehensive Cancer Network; ASCO, American Society of Clinical Oncology; SITC, Society for Immunotherapy of Cancer; BAL, bronchoalveolar lavage; DLCO, carbon monoxide diffusing capacity; CXR, chest X-ray; ADL, activities of daily living; CRP, C-reactive protein; MMF, Mycophenolate Mofetil; IVIG, Intravenous immunoglobulin.

was improved quickly. Paradoxically, the patient suffered from pneumonitis after only one cycle of camrelizumab administration. We surmised that EGFR inhibitors increased the risk of immune-associated pneumonitis (30). Was it possible that the administration of nimotuzumab caused the increased lung sensitivity?

Camrelizumab received approval in China in May 2019. For patients suffering from nasopharyngeal carcinoma, hepatocellular carcinoma, esophageal squamous cell carcinoma, gastric/gastroesophageal junction cancer and classical Hodgkin lymphoma, camrelizumab is effective (14,31-36). The incidence of IMP caused by camrelizumab is $1-6.7 \%(14,32,34-36)$. Most of patients with grade $1-2$ pneumonitis improved after treatment interruption. Only one died of pneumonitis. Some patients have basic lung diseases. It was reported that one patient who had received radiotherapy before developed radiation recall pneumonitis (RRP) after receiving camrelizumab. IMP also occurred in another two patients who administered camrelizumab combined with microwave ablation (37). Combined with this case, camrelizumab administration of radiotherapy, microwave ablation, and EGFR-TKI were the risk factors of pneumonitis. In terms of IMP patients, we are cautious about re-challenging the administration of camrelizumab. Some cases have more serious IMP due to the re-use of camrelizumab after the occurrence of IMP (33). Re- challenge needs to be evaluated by clinicians by combining with clinical effects and patients' specific conditions.

The disadvantage of the case is that due to the effect of COVID-19, treatment and examination were limited by some objective factors. Meanwhile, as the patient participated in a double-blind clinical study, it is unclear whether the patient received Tislelizumab.

\section{Conclusions}

In conclusion, we reported a case of IMP induced by antiPD-1 blockade camrelizumab 12 days after first dose. The patient has the history of smoking, radiotherapy, and combination medication that are all risk factors of IMP. In clinical practices, it is significant to attach close attentions to patients who have risk factors, including elderly male, smoking history, radiotherapy history, combination therapy, and basic lung disease. Lung radiological examination, timely corticosteroids intervention and drug withdrawal are key treatments.

\section{Acknowledgments}

We gratefully acknowledge the assistance of Beijing Friendship Hospital.

Funding: None. 


\section{Footnote}

Reporting Checklist: The authors have completed the CARE Reporting Checklist. Available at http://dx.doi. org/10.21037/apm-21-23

Conflicts of Interest: All authors have completed the ICMJE uniform disclosure form (available at http://dx.doi. org/10.21037/apm-21-23). The authors have no conflicts of interest to declare.

Ethical Statement: The authors are accountable for all aspects of the work in ensuring that questions related to the accuracy or integrity of any part of the work are appropriately investigated and resolved. All procedures performed in studies involving human participants were in accordance with the ethical standards of the institutional and/or national research committee(s) and with the Helsinki Declaration (as revised in 2013). Written informed consent was obtained from the patient.

Open Access Statement: This is an Open Access article distributed in accordance with the Creative Commons Attribution-NonCommercial-NoDerivs 4.0 International License (CC BY-NC-ND 4.0), which permits the noncommercial replication and distribution of the article with the strict proviso that no changes or edits are made and the original work is properly cited (including links to both the formal publication through the relevant DOI and the license). See: https://creativecommons.org/licenses/by-nc-nd/4.0/.

\section{References}

1. Baxi S, Yang A, Gennarelli RL, et al. Immune-related adverse events for anti-PD-1 and anti-PD-L1 drugs: systematic review and meta-analysis. Bmj 2018;360:k793.

2. De Velasco G, Je Y, Bossé D, et al. Comprehensive Metaanalysis of Key Immune-Related Adverse Events from CTLA-4 and PD-1/PD-L1 Inhibitors in Cancer Patients. Cancer Immunol Res 2017;5:312-8.

3. Zhang S, Liang F, Zhu J, et al. Risk of Pneumonitis Associated with Programmed Cell Death 1 Inhibitors in Cancer Patients: A Meta-analysis. Mol Cancer Ther 2017;16:1588-95.

4. Eun Y, Kim IY, Sun JM, et al. Risk factors for immunerelated adverse events associated with anti-PD-1 pembrolizumab. Sci Rep 2019;9:14039.

5. Wu J, Hong D, Zhang X, et al. PD-1 inhibitors increase the incidence and risk of pneumonitis in cancer patients in a dose-independent manner: a meta-analysis. Sci Rep 2017;7:44173.

6. Khunger M, Rakshit S, Pasupuleti V, et al. Incidence of Pneumonitis With Use of Programmed Death 1 and Programmed Death-Ligand 1 Inhibitors in Non-Small Cell Lung Cancer: A Systematic Review and MetaAnalysis of Trials. Chest 2017;152:271-81.

7. Huang Y, Fan H, Li N, et al. Risk of immune-related pneumonitis for PD1/PD-L1 inhibitors: Systematic review and network meta-analysis. Cancer Med 2019;8:2664-74.

8. Cui P, Liu Z, Wang G, et al. Risk factors for pneumonitis in patients treated with anti-programmed death-1 therapy: A case-control study. Cancer Med 2018;7:4115-20.

9. Teng F, Li M, Yu J. Radiation recall pneumonitis induced by PD-1/PD-L1 blockades: mechanisms and therapeutic implications. BMC Med 2020;18:275.

10. Sun Y, Shao C, Li S, et al. Programmed cell death 1 (PD1)/PD-ligand 1(PD-L1) inhibitors-related pneumonitis in patients with advanced non-small cell lung cancer. Asia Pac J Clin Oncol 2020;16:299-304.

11. Schoenfeld JD, Nishino M, Severgnini M, et al. Pneumonitis resulting from radiation and immune checkpoint blockade illustrates characteristic clinical, radiologic and circulating biomarker features. J Immunother Cancer 2019;7:112.

12. Chu X, Zhao J, Zhou J, et al. Association of baseline peripheral-blood eosinophil count with immune checkpoint inhibitor-related pneumonitis and clinical outcomes in patients with non-small cell lung cancer receiving immune checkpoint inhibitors. Lung Cancer 2020;150:76-82.

13. Markham A, Keam SJ. Camrelizumab: First Global Approval. Drugs 2019;79:1355-61.

14. Huang J, Xu B, Mo H, et al. Safety, Activity, and Biomarkers of SHR-1210, an Anti-PD-1 Antibody, for Patients with Advanced Esophageal Carcinoma. Clin Cancer Res 2018;24:1296-304.

15. Tirumani SH, Ramaiya NH, Keraliya A, et al. Radiographic Profiling of Immune-Related Adverse Events in Advanced Melanoma Patients Treated with Ipilimumab. Cancer Immunol Res 2015;3:1185-92.

16. Pozzessere C, Bouchaab H, Jumeau R, et al. Relationship between pneumonitis induced by immune checkpoint inhibitors and the underlying parenchymal status: a retrospective study. ERJ Open Res 2020;6:00165-2019.

17. Barrón F, Sánchez R, Arroyo-Hernández M, et al. Risk of Developing Checkpoint Immune Pneumonitis and Its 
Effect on Overall Survival in Non-small Cell Lung Cancer Patients Previously Treated With Radiotherapy. Front Oncol 2020;10:570233.

18. Sharpe AH, Pauken KE. The diverse functions of the PD1 inhibitory pathway. Nat Rev Immunol 2018;18:153-67.

19. Feng S, Coward J, McCaffrey E, et al. PembrolizumabInduced Encephalopathy: A Review of Neurological Toxicities with Immune Checkpoint Inhibitors. J Thorac Oncol 2017;12:1626-35.

20. Petri CR, Patell R, Batalini F, et al. Severe pulmonary toxicity from immune checkpoint inhibitor treated successfully with intravenous immunoglobulin: Case report and review of the literature. Respir Med Case Rep 2019;27:100834.

21. Mori H, Sakai C, Iwai M, et al. Immune thrombocytopenia induced by nivolumab in a patient with non-small cell lung cancer. Respir Med Case Rep 2019;28:100871.

22. Raskin J, Masrori P, Cant A, et al. Recurrent dysphasia due to nivolumab-induced encephalopathy with presence of Hu autoantibody. Lung Cancer 2017;109:74-7.

23. Zhao ZM, Liu SC, Xu XJ, et al. Treatment of Skin Reaction Induced by Nivolumab Combined with Radiotherapy in Non-small Cell Lung Cancer: A Case Report. Chin Med Sci J 2018;33:183-7.

24. Naidoo J, Wang X, Woo KM, et al. Pneumonitis in Patients Treated With Anti-Programmed Death-1/ Programmed Death Ligand 1 Therapy. J Clin Oncol 2017;35:709-17.

25. Nishino M, Ramaiya NH, Awad MM, et al. PD-1 Inhibitor-Related Pneumonitis in Advanced Cancer Patients: Radiographic Patterns and Clinical Course. Clin Cancer Res 2016;22:6051-60.

26. Haanen JBAG, Carbonnel F, Robert C, et al. Management of toxicities from immunotherapy: ESMO Clinical Practice Guidelines for diagnosis, treatment and followup. Ann Oncol 2017;28:iv119-42.

27. Thompson JA, Schneider BJ, Brahmer J, et al. NCCN Guidelines Insights: Management of ImmunotherapyRelated Toxicities, Version 1.2020. J Natl Compr Canc Netw 2020;18:230-41.

28. Brahmer JR, Lacchetti C, Schneider BJ, et al. Management of Immune-Related Adverse Events in Patients Treated
With Immune Checkpoint Inhibitor Therapy: American Society of Clinical Oncology Clinical Practice Guideline. J Clin Oncol 2018;36:1714-68.

29. Puzanov I, Diab A, Abdallah K, et al. Managing toxicities associated with immune checkpoint inhibitors: consensus recommendations from the Society for Immunotherapy of Cancer (SITC) Toxicity Management Working Group. J Immunother Cancer 2017;5:95.

30. Oshima Y, Tanimoto T, Yuji K, et al. EGFR-TKIAssociated Interstitial Pneumonitis in Nivolumab-Treated Patients With Non-Small Cell Lung Cancer. JAMA Oncol 2018;4:1112-5.

31. Xu J, Zhang Y, Jia R, et al. Anti-PD-1 Antibody SHR-1210 Combined with Apatinib for Advanced Hepatocellular Carcinoma, Gastric, or Esophagogastric Junction Cancer: An Open-label, Dose Escalation and Expansion Study. Clin Cancer Res 2019;25:515-23.

32. Fang W, Yang Y, Ma Y, et al. camrelizumab (SHR-1210) alone or in combination with gemcitabine plus cisplatin for nasopharyngeal carcinoma: results from two single-arm, phase 1 trials. Lancet Oncol 2018;19:1338-50.

33. Qin S, Ren Z, Meng Z, et al. Camrelizumab in patients with previously treated advanced hepatocellular carcinoma: a multicentre, open-label, parallel-group, randomised, phase 2 trial. Lancet Oncol 2020;21:571-80.

34. Nie J, Wang C, Liu Y, et al. Addition of Low-Dose Decitabine to Anti-PD-1 Antibody camrelizumab in Relapsed/Refractory Classical Hodgkin Lymphoma. J Clin Oncol 2019;37:1479-89.

35. Huang J, Xu J, Chen Y, et al. Camrelizumab versus investigator's choice of chemotherapy as second-line therapy for advanced or metastatic oesophageal squamous cell carcinoma (ESCORT): a multicentre, randomised, open-label, phase 3 study. Lancet Oncol 2020;21:832-42.

36. Song $\mathrm{Y}, \mathrm{Wu} \mathrm{J}$, Chen $\mathrm{X}$, et al. A Single-Arm, Multicenter, Phase II Study of camrelizumab in Relapsed or Refractory Classical Hodgkin Lymphoma. Clin Cancer Res 2019;25:7363-9.

37. Wei Z, Yang X, Ye X. Rechallenge of camrelizumab in non-small-cell lung cancer patients treated previously with camrelizumab and microwave ablation. J Cancer Res Ther 2020;16:1191-5.
Cite this article as: Li L, Lou A, Yu J. Immune checkpoint inhibitor-related pneumonitis induced by camrelizumab: a case report and review of literature. Ann Palliat Med 2021;10(7):84608466. doi: 10.21037/apm-21-23 